\title{
On the Design and Measurement of a Novel Non- Orthogonal Multi-Beam Network for Triangular Arrays of Three Radiating Elements
}

\author{
Javier García-Gasco Trujillo, Álvaro Noval Sánchez de Toca, Ignacio Montesinos-Ortego, Manuel Sierra Perez \\ Radiation Group, Systems and Radiocommunications Department \\ Technical University of Madrid \\ Ciudad Universitaria, Madrid, Spain \\ \{jtrujillo, anoval, nacho,m.sierra.perez\}@gr.ssr.upm.es
}

\begin{abstract}
An innovative dissipative multi-beam network for triangular arrays of three radiating elements is proposed. This novel network provides three orthogonal beams in $\theta_{0}$ elevation angle and a fourth one in the broadside steering direction. The network is composed of $90^{\circ}$ hybrid couplers and fixed phase shifters. In this paper, a relation between network components, radiating element distance and beam steering directions will be shown. Application of the proposed dissipative network to the triangular cells of three radiating elements that integrate the intelligent antenna GEODA will be exhibited. This system works at $1.7 \mathrm{GHz}$, it has a $60^{\circ}$ single radiating element beamwidth and a distance between array elements of $0.57 \lambda$. Both beam patterns, theoretical and simulated, obtained with the network will be depicted. Moreover, the whole system, dissipative network built with GEODA cell array, has been measured in the anechoic chamber of the Radiation Group of Technical University of Madrid, demonstrating expected performance.
\end{abstract}

Butler matrix; phased arrays; adaptive arrays; nonlinear network analysis; multi-beam forming networks (key words)

\section{INTRODUCTION}

From the beginning of telemetry, tracking and command (TT\&C) systems, mechanical scanning antennas have been employed at ground stations. As it is well-known, this technique is not only sensitive to gravity and mechanical failure but also slow, making simultaneous satellite communications infeasible. The use of electronically scanned antenna arrays overcomes these limitations, allowing much faster multi-beam scans without physical antenna rotation, $[1,2]$. These types of systems that use information from the link environment to set the beam shape are called intelligent architectures [3].

In order to improve the ground station performance, many studies have been carried out in the field of electronic scanning systems, in which large arrays composed of thousand of radiating elements have been considered. Generally, these array structures are divided into subarrays based on cells of at least three radiating elements [4-6]. This segmentation reduces the total number of active circuits required, such as amplifiers or phase shifters [7], decreasing total cost and improving efficiency. However, this simplification may imply short restriction in the beam steering precision control that must be taken into account [8]. Electronic steering technique is based on the control of the relative phase associated to each antenna that composes the whole array. The relative phase can be modified by using signal processing algorithms, e.g. MUSIC [9] or ESPRIT [10], or placing phase shifter circuits inside the hardware antenna.

Nowadays, software/hardware hybrid architectures are gaining special interest because its versatility. Therefore, the study of structures that provides multiple beams in different spatial range associated to each cell is needed. In this paper, a new multibeam network configuration that provides three orthogonal beams in $\theta_{0}$ elevation angle and an extra one with broadside steering direction by feeding a triangular array of three radiating elements will be introduced. Section II will present a short introduction to the state of art of classical multibeam networks [11, 12]. Section III will comment lossless networks analysis, including two original lossless networks designs [13]. Section IV will show general dissipative matrices theory as well as many applications for array antennas of three radiating elements. In Section $V$ final multi-beam network proposed will be simulated, built and measured in a practical case. Finally, Section VI will collect the conclusion drawn during the paper.

\section{GENERAL MBFM SCHEME}

One of the most important tasks in a phased array antenna is designing the beam forming network (BFN). A BFN essentially provides the necessary amplitudes and phases to the radiating elements to produce the desired beams. In particular, for a multiple-beam array, an appropriate $\mathrm{BFN}$ is vital in order to distribute the signals to the radiating ports properly.

Most common types of BFNs are presented in [7], where Butler matrix, Blass network, Rotman lens and many more are explained. Some of the designs studied in this paper are based on Butler matrix modifications. Butler matrix is employed to produce $2^{n}$ orthogonal beams in linear arrays composed of $2^{n}$ radiating elements, where $n$ is a positive integer. Changing some configuration details it is possible to break the limits of this useful network, as will be shown later. 
BFNs behavior is characterized by its scattering matrix, where each matrix element represents the mutual coupling between each input and output. Considering inputs and outputs adapted and isolated from each other, an $\mathrm{MxN}$ network responds to the scattering matrix defined in (1),

$[S]=\left[\begin{array}{cccccc}0 & \cdots & 0 & S_{1, M+1} & \cdots & S_{1, M+N} \\ \vdots & \ddots & \vdots & \vdots & \ddots & \vdots \\ 0 & \cdots & 0 & S_{N, M+1} & \cdots & S_{N, M+N} \\ S_{N+1,1} & \cdots & S_{N+1, M} & 0 & \cdots & 0 \\ \vdots & \ddots & \vdots & \vdots & \ddots & \vdots \\ S_{N+M, 1} & \cdots & S_{N+M, M} & 0 & \cdots & 0\end{array}\right]=\left[\begin{array}{cc}0 & S_{R} \\ S_{T} & 0\end{array}\right](1)$ where $S_{T}$ equals $S_{R}^{H}$ if the network is reciprocal.

\section{LOSSLESS NETWORK FOR TRIANGULAR ARRAYS}

When a network is reciprocal and lossless, the product of the scattering matrix and its transpose conjugate is the identity matrix,

$$
[S][S]^{H}=[I]
$$

This implies that vectors related to the columns of each matrix $S_{T}$ and $S_{R}$ have to be orthonormal to each. Hence,

- The number of input and outputs must be the same. Otherwise, matrices would be non-square matrices and one of them would have more vectors than matrix range wherewith it would be impossible to obtain orthogonal beams to each.

- Unitary excitations indicate that the output power is equal to the input power.

- Vectors are orthogonal. When an input port is excited, the radiation pattern obtained will be orthogonal to any other one generated by any other input port.

\section{A. Basic Equations}

In order to analyze a triangular 'cell', it is assumed patches are located over vertices of an equilateral triangle with $d$ side length. Figure 1 shows a scheme where: $x_{1}=\frac{d}{2 \sqrt{3}}, y_{1}=\frac{d}{2}$; $x_{2}=\frac{d}{2 \sqrt{3}}, y_{2}=-\frac{d}{2} ; x_{3}=-\frac{d}{\sqrt{3}}, y_{3}=0$.

Considering the steering direction of the first beam as $\theta=\theta_{0}, \phi=0$, and taking into account that the array factor is given by,

$$
A F(\theta, \phi)=\sum_{i=1}^{3} A_{i} e^{-j \alpha_{i}} e^{-j k \hat{n}_{i}}
$$

Contemplating the fact that feeding phases must satisfy the condition of adding the contributions of each array element in the steering direction, then,

$$
k_{0} \hat{r} r_{1}-\alpha_{1}=k_{0} \hat{r} r_{2}-\alpha_{2}=k_{0} \hat{r} r_{3}-\alpha_{3} .
$$

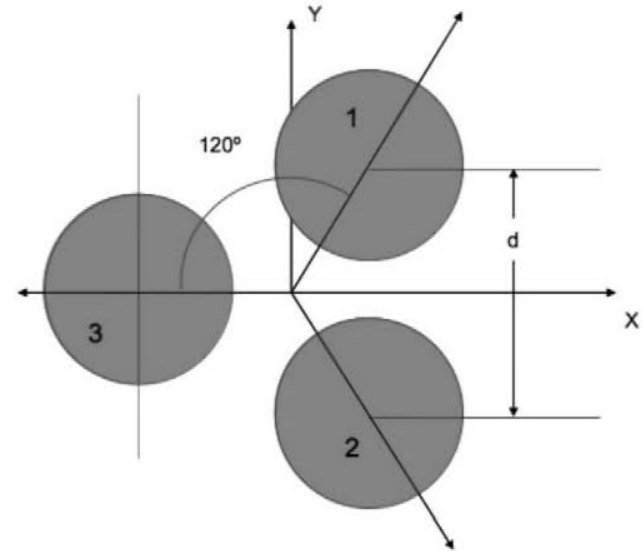

Fig.1: Subarray distribution

Hence, it is shown that,

$$
\begin{gathered}
S_{4,1}=S_{5,1}=a e^{j \alpha} \\
S_{6,1}=b e^{j(\alpha-\beta)}
\end{gathered}
$$

where,

$$
\beta=\sqrt{3} \frac{\pi d}{\lambda} \sin \left(\theta_{0}\right)
$$

Rotating $S$ parameters, (5.1) and (5.2), three different beams are obtained. The other two beams will be orthogonal, keeping a rotational symmetry with the same elevation angle and $\pm 120^{\circ}$ related to the azimuth angle.

Imposing the orthogonal condition, (2), ensured through mutually orthogonal excitation vectors at the array port,

$$
\sin \left(\theta_{0}\right)=\frac{\lambda}{\sqrt{3} \pi d} \arccos \left(-\frac{a}{2 b}\right)
$$

Main steering directions $\theta_{0}$, depends on both, distance and

\begin{tabular}{|c|c|c|c|c|c|}
\hline & & \multicolumn{4}{|c|}{$d / \lambda$} \\
\hline & & 0.5 & 0.6 & 0.7 & 0.8 \\
\hline $\mathbf{a} / \mathbf{b}$ & $\beta$ & $\boldsymbol{\theta}_{0}$ & $\boldsymbol{\theta}_{0}$ & $\boldsymbol{\theta}_{0}$ & $\boldsymbol{\theta}_{0}$ \\
\hline 0.5 & 104.5 & 42.1 & 34.0 & 28.6 & 24.8 \\
\hline 0.7 & 110.5 & 45.1 & 36.2 & 30.4 & 26.3 \\
\hline 1.0 & 120.0 & 50.3 & 39.9 & 33.4 & 28.8 \\
\hline 1.3 & 130.5 & 56.9 & 44.3 & 36.7 & 31.6 \\
\hline 1.5 & 138.6 & 62.8 & 47.8 & 39.4 & 33.8 \\
\hline
\end{tabular}
feeding signal relation, between elements. Table I shows array factor $\theta_{0}$ for different distances between elements $d$, and different amplitude/phase feeding relation $a / b$, (note: it does not take into account the radiation pattern of the single radiating element).

TABLE I. ARRAY FACTOR STEERING DIRECTION

Should be noted that when the feeding amplitude is the same for the three elements then it is needed a $120^{\circ}$ feeding phase shifts $\beta$. 


\section{B. Lossless Network Designs}

Once analysis has been performed, the hard task is to find structures that fulfill the desired behavior. Different $3 \times 3$ MBFN schemes based on hybrid couplers and fixed phase shifter has been studied, [13]. Figure 2 (a) shows a scheme based on Butler network that provides uniform amplitude $a / b$ equals 1 with $\beta$ equals $120^{\circ}$. Figure 2 (b) depicted a rotation symmetric scheme that offers a perfect symmetrical behavior between inputs and outputs. Intrinsic features of these networks limit the number of beams provided at the number of radiating elements and impose the orthogonally between those beams. This analysis shows the need for a beam in the broadside direction. Therefore, this research leads to consider the possibility of using dissipative networks, which are capable of providing a higher number of beams than the radiating elements compounding the cell.

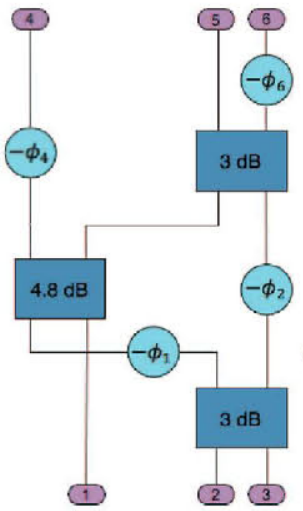

(a)

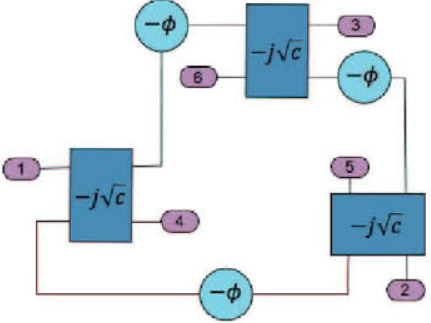

(b)
Fig. 2: (a) Scheme of a three port modified Butler network. (b) Scheme of a three port symmetric network

\section{DISSIPATIVE NETWORK FOR TRIANGULAR ARRAYS}

Because of these networks have losses, there is no need to accomplish beams orthogonal condition, which gives a greater degree of freedom that could be used to control beam steering directions. It is worth noting the orthogonal condition ensures maximum array gain. However, when radiating elements are related to radiofrequency circuits in which amplifiers are found, the relevant parameter is the factor $G / T$ that shows the quality of the antenna. As a result, there is no problem on using dissipative network whenever there is an amplifier stage.

The scattering matrix associated to the dissipative network under study, which has three orthogonal beams in $\theta_{0}$ (columns 2 to 4 ) and an additional beam in the broadside direction (column 1), is as follows,

$$
\left[S_{T}\right]=\left[\begin{array}{cccc}
f & b e^{-j \beta} & a & a \\
f & a & b e^{-j \beta} & a \\
f & a & a & b e^{-j \beta} \\
g_{1} e^{j \alpha_{1}} & g_{2} e^{j \alpha_{2}} & g_{3} e^{j \alpha_{3}} & g_{4} e^{j \alpha_{4}}
\end{array}\right] .
$$

The steering direction of the three orthogonal beams depends on the feeding factors, $(b, c)$, and on the distance between elements of the array, $(d)$, and it is giving by,

$$
\sin \left(\theta_{0}\right)=\frac{\lambda}{\sqrt{3} \pi d} \cos ^{-1}\left(\frac{a^{2}+b^{2}-1}{2 a b}\right) .
$$

Losses associated with the network are related to the broadside beam power as,

$$
\begin{gathered}
g_{1}=1-3 f^{2} \\
g_{i}=f^{2} \text { con } i=2,3,4
\end{gathered}
$$

Several dissipative networks for triangular arrays have been studied. The most common difficult, is to avoid the appearance of side lobes and diffraction, as shown in [13], where the truncated Butler network for three radiating elements shown in Fig. 3 was presented and analyzed.

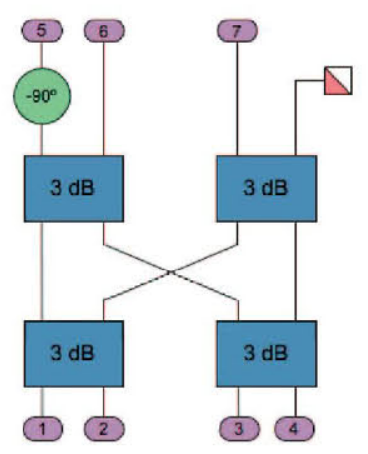

Fig. 3: Scheme of a $4 \times 3$ modify Butler matrix

Finally, a network as shown in Fig. 4, is proposed. This network is composed of four $3 \mathrm{~dB} 90^{\circ}$ couplers, four fixed phase shifters and an additional $20 \log (\sqrt{c}) \mathrm{dB} 90^{\circ}$ coupler. Its scattering matrix $S_{T}$ is given by,

$$
\begin{aligned}
& {\left[S_{T}\right]=} \\
& \frac{1}{2}\left[\begin{array}{cccc}
e^{-j \alpha}-\sqrt{1-c} & -j\left(e^{-j \alpha}+\sqrt{1-c}\right) & -\sqrt{c} & j \sqrt{c} \\
-j\left(e^{-j \alpha}+\sqrt{1-c}\right) & -\left(e^{-j \alpha}-\sqrt{1-c}\right) & -j \sqrt{c} & -\sqrt{c} \\
-\sqrt{c} e^{-j \phi_{s}} & -j \sqrt{c} e^{-j \phi_{s}} & \left(-\left(e^{-j \alpha}-\sqrt{1-c}\right)\right) e^{-j \phi_{s}} & \left(-j\left(e^{-j \alpha}+\sqrt{1-c}\right)\right) e^{-j \phi_{s}} \\
j \sqrt{c} e^{-j \phi_{\alpha}} & -\sqrt{c} e^{-j \phi_{\alpha}} & \left(-j\left(e^{-j \alpha}+\sqrt{1-c}\right)\right) e^{-j \phi_{4}} & \left(e^{-j \alpha}-\sqrt{1-c}\right) e^{-j \phi_{\alpha}}
\end{array}\right]
\end{aligned}
$$

The analysis of the scattering parameters of the network shows the relation between components. This analysis seeks to match the scattering matrix associated to the dissipative network proposed, (10), to the desired pattern scattering matrix, (6). It shows the behavior of the network is governed by the value of the central coupler, which determines the phase shifters needed. The relation between the steering direction angle $\theta_{0}$ and the phase shift between elements $\beta$ is 
given by,

$$
\beta=\sqrt{3} \pi \frac{d}{\lambda} \sin \left(\theta_{0}\right)
$$

The steering direction of the orthogonal beams as well as losses associated with each of the beams depends on the coupler $c$ as,

$$
\begin{aligned}
\sin \left(\theta_{0}\right) & =\tan ^{-1}\left(\frac{-2 \sqrt{1-c}}{-\sqrt{c}}\right) \frac{1}{\sqrt{3} \pi} \frac{\lambda}{d} \\
L_{\theta_{0}} & =-10 \log \left(1-\frac{c}{4}\right) \\
L_{\theta_{0}} & =-10 \log \left(\frac{3}{4} c\right)
\end{aligned}
$$

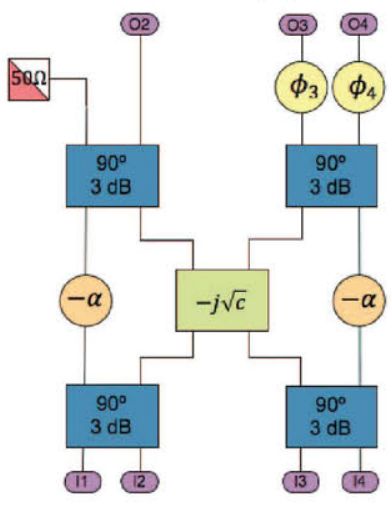

Fig. 4: $4 \times 3$ dissipative network

Table II shows beam steering directions $\theta_{0}$ and distributed signal amplitudes and phases depending on the parameters $c$, $\alpha$ and $d$. Losses introduced by the network are also calculated, both $\theta_{0}$ beams steering direction, $L_{\theta_{0}}$, and

\begin{tabular}{|c|c|c|c|c|c|c|c|c|}
\hline \multirow[b]{2}{*}{$c$} & \multirow[b]{2}{*}{$a$} & \multirow[b]{2}{*}{$\bar{b}$} & \multirow[b]{2}{*}{$\beta\left[^{\circ}\right]$} & \multirow[b]{2}{*}{$\alpha$} & \multirow[b]{2}{*}{0.5} & \multicolumn{3}{|c|}{$\theta_{0}$ parad $/ \lambda\left[^{\circ}\right]$} \\
\hline & & & & & & 0.6 & 0.7 & 0.8 \\
\hline 0.00 & 0.000 & 1.000 & -90.0 & 0.0 & -35.3 & -28.8 & -24.4 & -21.2 \\
\hline 0.10 & 0.025 & 0.925 & -99.5 & 18.4 & -39.6 & -32.1 & -27.1 & -23.5 \\
\hline 0.20 & 0.050 & 0.850 & -104.0 & 26.6 & -41.9 & -33.8 & -28.5 & -24.7 \\
\hline 0.30 & 0.075 & 0.775 & -108.1 & 33.2 & -43.9 & -35.3 & -29.7 & -25.7 \\
\hline 0.40 & 0.100 & 0.700 & -112.2 & 39.2 & -46.0 & -36.9 & -31.0 & -26.7 \\
\hline 0.50 & 0.125 & 0.625 & -116.6 & 45.0 & -48.4 & -38.6 & -32.3 & -27.9 \\
\hline 0.60 & 0.150 & 0.550 & -121.4 & 50.8 & -51.2 & -40.5 & -33.8 & -29.2 \\
\hline 0.70 & 0.175 & 0.475 & -127 & 56.8 & -54.8 & -42.9 & -35.7 & -30.7 \\
\hline 0.80 & 0.200 & 0.400 & -135.0 & 63.4 & -60.0 & -46.2 & -38.2 & -32.8 \\
\hline 0.90 & 0.225 & 0.325 & -146.3 & 71.6 & -69.8 & -51.5 & -42.1 & -36.0 \\
\hline 1.00 & 0.250 & 0.250 & -180.0 & 90.0 & -- & -74.2 & -55.6 & -46.2 \\
\hline
\end{tabular}
broadside beams, $L_{0^{\circ}}$, Table III.

TABLE II. STEERING DIRECTION DEPENDING ON PARAMETERS C AND DISTANCES BETWEEN RADIATING ELEMENTS D

TABLE III. LOSSES NETWORK RELATED TO $C$ COUPLING

\begin{tabular}{c|c|c}
\hline $\boldsymbol{c}$ & $\boldsymbol{L}_{\boldsymbol{\theta}}[\mathrm{dB}]$ & $\boldsymbol{L}_{0}[\mathbf{d B}]$ \\
\hline 0.00 & 0.00 & $\infty$ \\
\hline 0.10 & 0.11 & 11.3 \\
\hline 0.20 & 0.22 & 8.24 \\
\hline 0.30 & 0.34 & 6.48 \\
\hline 0.40 & 0.46 & 5.23 \\
\hline 0.50 & 0.58 & 4.26 \\
\hline 0.60 & 0.71 & 3.47 \\
\hline 0.70 & 0.84 & 2.80 \\
\hline 0.80 & 0.97 & 2.22 \\
\hline 0.90 & 1.11 & 1.71 \\
\hline 1.00 & 1.25 & 1.25 \\
\hline
\end{tabular}

\section{IMPLEMENTATION OF THE PROPOSED 4X3 NETWORK}

Microstrip application of the proposed dissipative network to the triangular cells of three radiating elements that integer the intelligent antenna GEODA, which is composed of 2.700 radiating elements working at $1.7 \mathrm{GHz}$ with a $60^{\circ}$ single radiating element beamwidth and a distance between array elements $d$ of $0.57 \lambda[6,14,15]$, is exhibited in this section.

Looking for a simple and small size implementation, a practical network could be given by using $c$ equals 0.5 . In this case, all $90^{\circ}$ hybrid couplers utilized are $3 \mathrm{~dB}$ and they all can be implemented easily by using commercial $3 \mathrm{~dB} 90^{\circ}$ hybrid couplers, such as Mini Circuits QCN-25. Phase shifters are obtained by additional fixed length lines when is required. The scattering matrix associated to this network, obtained by replacing matrix (10), will be,

$\left|S_{T}\right|=\left[\begin{array}{cccc}0.353 & 0.791 & 0 & 0.353 \\ 0.791 & 0.353 & 0.353 & 0.353 \\ 0.353 & 0.353 & 0.353 & 0.791 \\ 0.353 & 0.353 & 0.791 & 0.353\end{array}\right] \arg \left[S_{T}\right]=\left[\begin{array}{cccc}-90^{\circ} & -116.6^{\circ} & -180^{\circ} & -270^{\circ} \\ -116.6^{\circ} & 90^{\circ} & -90^{\circ} & -180^{\circ} \\ 0^{\circ} & 90^{\circ} & -90^{\circ} & 63^{\circ} \\ 0^{\circ} & 90^{\circ} & -206.6^{\circ} & -180^{\circ}\end{array}\right]$

The radiating element used consists of two stacked circular patches. As it is shown in [13], the bandwidth of its radiation pattern is on the order of $60^{\circ}$.

With the aim of checking a correct performance, the ideal network has been simulated by utilizing Advanced Design System (ADS) 2009, Fig. 5. The scattering matrix shows a perfect behavior. Once the ideal architecture shows a good performance, the real network is simulated by using measured $s$ parameters of both, phase shifters and QCN-25 $90^{\circ} 3 \mathrm{~dB}$ hybrid coupler, showing agreement with expectations.
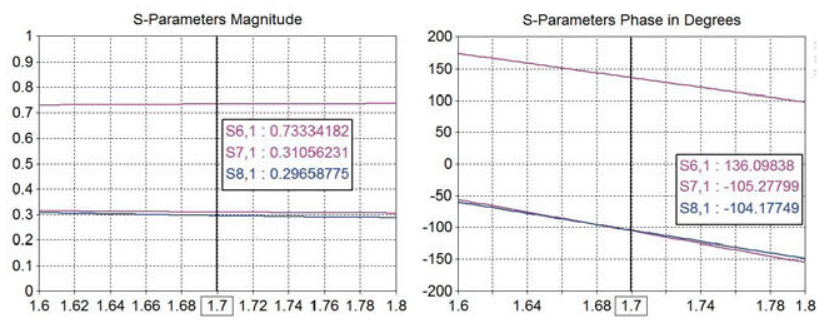

(a)
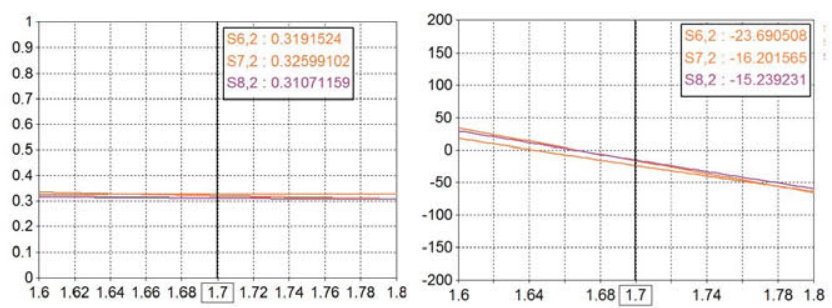

(b)

Fig. 5: Simulated scattering parameters of the real dissipative $4 \times 3$ network. (a) $\theta_{0}$ tilt beam, and (b) broadside beam. 
The network has been implemented in microstrip RO4350B, Fig. 6. Fixed phase shifters and $3 \mathrm{~dB} 90^{\circ}$ hybrid couplers QCN-25 have been evaluated independently in order to assure a good performance. The use of commercial couplers is important when total size dimensions must be minimized. The network has $\pm 0.3 \mathrm{~dB}$ module error and $\pm 5^{\circ}$ phase error, which is acceptable, as it is depicted in Fig. 7 where a comparison between radiation patterns simulated with theoretical and measured $s$ parameters shows low beam deviations.

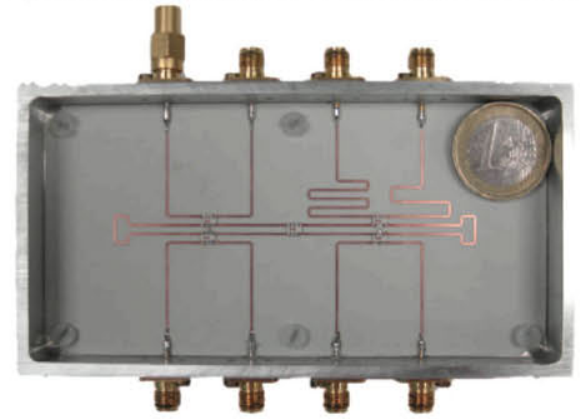

Fig. 6: Microstrip non-orthogonal network

TABLE IV. SCATTERING MATRIX AND LOSSES OF THE 4X3 NETWORK

\begin{tabular}{cccccc} 
& \multicolumn{6}{c}{$\mathbf{0 2}$} & $\mathbf{O 3}$ & $\mathbf{O 4}$ & $\boldsymbol{\beta}$ & Losses \\
\hline I1 & $0.700 e^{j 0.915 \pi}$ & $0.305 e^{-j 0.446 \pi}$ & $0.302 e^{-j 0.412 \pi}$ & $118.1^{\circ} \pm 3^{\circ}$ & $32 \%$ \\
\hline I2 & $0.327 e^{j 0.032 \pi}$ & $0.312 e^{j 0.049 \pi}$ & $0.300 e^{j 0.068 \pi}$ & $0^{\circ} \pm 3^{\circ}$ & $70 \%$ \\
\hline I3 & $0.312 e^{-j 0.951 \pi}$ & $0.327 e^{-j 0.969 \pi}$ & $0.683 e^{j 0.417 \pi}$ & $112.1^{\circ} \pm 2^{\circ}$ & $33 \%$ \\
\hline I4 & $0.312 e^{j 0.537 \pi}$ & $0.691 e^{-j 0.108 \pi}$ & $0.324 e^{j 0.572 \pi}$ & $119.1^{\circ} \pm 3^{\circ}$ & $32 \%$ \\
\hline
\end{tabular}

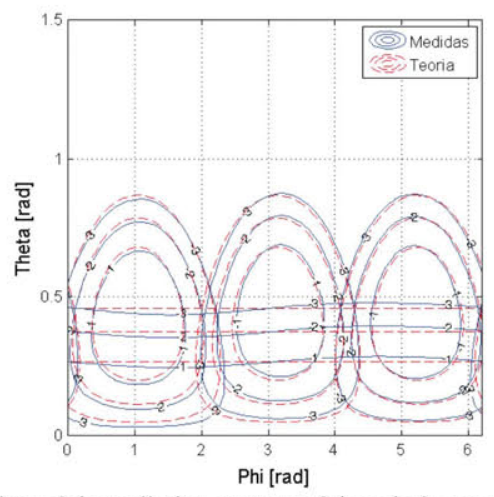

Fig. 7: Simulation of the radiation pattern of the whole system, cell antenna and dissipative network, considering theoretical (red) and measured (blue) $s$ parameters

Moreover, the whole system, dissipative network built with GEODA cell array, has been measured in the anechoic chamber of the Radiation Group of Technical University of Madrid, demonstrating expected performance (Fig. 8).
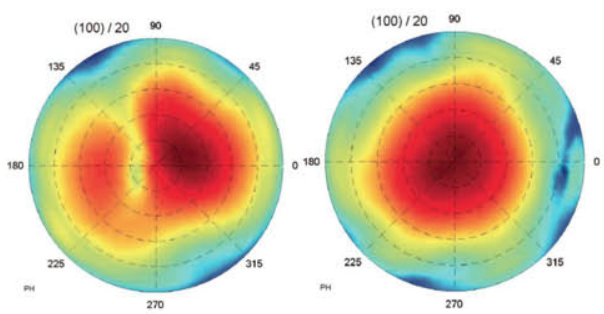
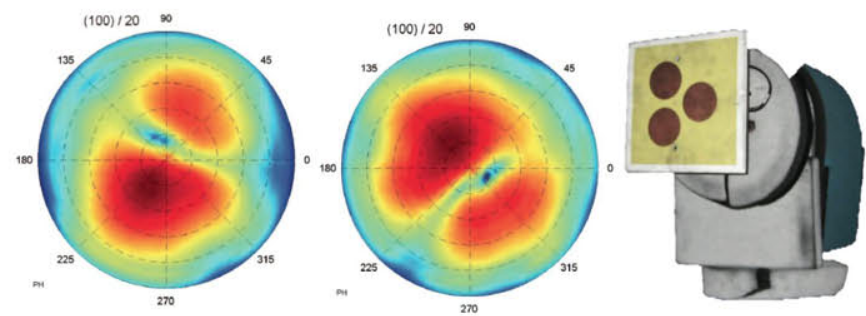

Fig. 8: Measured radiation patterns of the beam forming network and cell subarray GEODA.

\section{CONCLUSIONS}

MBFN for triangular subarrays of three radiating elements has been studied. Intrinsic features of lossless network limit the number of beams generated. A novel non-orthogonal network providing three ortogonal $\theta_{0}$ beams and an extra broadside one has being proposed. Practical implementation of the network integrated to GEODA triangular subarray has been built and measured obtaining expected results.

\section{ACKNOWLEDGMENT}

This work is been supported by CROCANTE project with reference TEC2008-06736-C03-01 and SICOMORO project with reference TEC-2011-28789-C02-01.

\section{REFERENCES}

[1] A. Torre, J. Gonzalo, M. Pulido, and R. Martínez Rodríguez-Osorio. "New generation Ground Segment Architecture for LEO satellites," 57th International Astronautical Congress, pp. 221-226. Valencia, Spain, October 2006.

[2] Constantine A. Balanis. Modern Antenna Handbook. John Wiley \& Sons, Inc. USA, 2008.

[3] L. C. Godara. "Smart Antennas $\left(1^{\text {st }}\right.$ Ed.)." CRC Press.

[4] B. Tomasic, J. Turtle, and S. Liu. "A Geodesic Sphere Phased Array Antenna for Satellite Control and Communication," XXVII General Assembly of the International union of Radio Science, Maastricht, August 2002.

[5] S. Liu, B. Tomasic, S. Hwang, and J. Turtle. "The Geodesic Dome Phased Array Antenna (GDPAA) for Satellite Operations Support. IEEE $18^{\text {th }}$ International Conference on Applied Electromagnetics and Communications, pp. 1-1, Dubrovnik, April 2006.

[6] I. Montesinos, M. Sierra Pérez, J. L. Fernández, R. Matínez, and J. L. Masa. "GEODA: Adaptive Antenna of Multiple Planar Arrays for Satellite Communicatinos," European Conference on Antenna and Propagation, Berlin, Germany 2009.

[7] Arun K. Bhattacharyya. "Phased Array Antennas." Wiley.

[8] Amir I. Zaghloul, Ozlem Kilic. "Distributed Beam Former for DistributedAperture Electronically Steered Antennas," $25^{\text {th }}$ Army Science Conference, Orlando, November 2006

[9] R. O. SCHMIDT, "Multiple Emitter Location and Signal Parameter Estimation," Proceedings of RADC Spectrum Estimation Workshop, Griffiss AFB, New York, pp. 243-258, 1979.

[10] R. H. Roy and T. Kailath, "ESPRIT-Estimation of Signal Parameters Via Rotational Invariance Techniques," IEEE Trans. Acoust., Speech, signal Process., Vol. 37, pp. 984-995, July 1989.

[11] R. C. Johnson, and H. Jasik, Antenna Engineering Handbook, McGraw-Hill, New York, 1984.

[12] R. Hansen, Phased Array Antennas, John Willey \& Sons, Hoboken, NJ, 1998.

[13] J. García-Gasco Trujillo; M. Sierra Pérez; A. Novo García; M. Vera-Isasa. "Multibeam Network Design and Measurement for Triangular Array of Three Radiationg Elements," 5th European Conference on Antennas and Propagation, Roma, Italia, Abril 2011.

[14] Miguel Alejandro Salas Natera, Andrés García Aguilar, Jonathan Mora Cueva, José Manuel Fernández, Pablo Padilla De La Torre, Javier García-Gasco Trujillo, Ramón Martínez Rodríguez-Osorio, Manuel Sierra-Perez, Leandro De Haro Ariet and Manuel Sierra Castañer (2011). New Antenna Array Architectures for Satellite Communications, Advances in Satellite Communications, Masoumeh Karimi and Yuri Labrador (Ed.), ISBN: 978-953-307-562-4, InTech.

[15] J. García-Gasco Trujillo, M. Salas Natera, I. Montesinos, M. Arias Campo, M. Sierra Pérez, R. Martínez, "GEODA-GRUA: Adaptive Multibeam Conformal Antenna for Satellites Communications," 30th URSI General Asembly and Scientific Sumposium of International Union of Radio Science, Estambul, Turquia, Agosto 2011. 